\title{
EL DEBATE SOBRE LA INEVITABILIDAD DEL ESTADO: NOTAS CRÍTICAS SOBRE LOS ARGUMENTOS DE RANDALL HOLCOMBE
}

\author{
MIGUEL ANXO BASTOS BOUBETA \\ ÓSCAR RODRÍGUEZ CARREIRO
}

Fecha de recepción: 23 de julio de 2020

Fecha de aceptación: 8 de septiembre de 2020

Resumen: Este trabajo consiste en análisis de los argumentos usados por Randall Holcombe para justificar la inebitalidad de la existencia de un ente monopolista de servicios de defensa y seguridad. Entendemos que su justificación es incompleta porque su definición de la necesidad de monopolio en la prestación de servicios de este tipo no se ajusta a la definción austríaca de bienes públicos o monopolio. Además no tiene en cuenta evidencias históricas de la prestación de dichos servicios en régimen de competencia.

Palabras clave: Estado, defensa, seguridad, monopolio, bienes públicos.

Clasificación JEL: B53, H41, L33, H44

Abstract: This work consists on an analysis of the arguments used by Randall Holcombe to justify the inevitability of the existence of a monopoly on the services of defence and security. We understand that his justification is incomplete because the definition that he uses, regarding the need of a monopoly in the provision of services of this type, does not fit with the Austrian definition of public goods or monopoly. Besides, it does not take into account historical evidence of the provision of these services in competition.

Keywords: State, defence, security, monopoly, public goods.

JEL Classification: B53, H41, L33, H44 


\section{INTRODUCCIÓN}

No cabe duda de que Randall G. Holcombe ha hecho grandes contribuciones a la hora de relativizar el papel del estado en su función de suministrar determinados bienes públicos, algunos de ellos consustanciales a su propia existencia, como la defensa (Holcombe, 2008), o a resaltar las consecuencias negativas de la intervención estatal en numerosos ámbitos de la vida social (Holcombe, 1995). Conoce bien la lógica de actuación de los gobiernos (Holcombe, 2002) y se ha mostrado siempre muy crítico con su desempeño. Sin embargo, hace unos años publicó un artículo (Holcombe, 2004) seguido de una secuela (Holcombe 2005) en las que pese a reconocer el carácter predatorio y no contractual de los estados se decanta, primero por apostar por su inevitabilidad, aun partiendo de una situación de anarquía, y segundo, dado que el estado es ineludible, por reclamar la creación de forma preventiva de una institución análoga pero que parta de una situación tal que le impida desarrollar su potencial predador a través de límites institucionales, al estilo de las diseñadas en el momento fundacional de los Estados Unidos. Dado que este artículo sigue despertando polémica y dado que se ha convertido con el tiempo en una de las principales referencias teóricas de los pensadores minarquistas, queremos en este trabajo criticar su proposición principal, esto es, que el estado es inevitable, y con ella, sus proposiciones auxiliares. Obviamente, estamos de acuerdo con su descripción de la lógica predadora del estado y con sus afirmaciones relativas al origen violento del mismo y a que pudiera ser justificado por el suministro de bienes públicos. También, como asociado que es a la escuela de la elección pública, asume una visión no benevolente de la actuación de los políticos, suponiéndoles una actuación egoísta e interesada, sea cual fuere el interés (tan egoísta puede ser la búsqueda de rentas como la imposición a la sociedad de determinados valores de los que gustamos). El resto de este artículo se centrará en diseccionar y discutir aquellos aspectos con los que discrepamos, sin, como hemos dicho, tratar de desmerecer para nada la calidad de su argumentación. 
II

\section{SOBRE LAS AGENCIAS DE PROTECCIÓN Y SU SUPUESTO MONOPOLIO}

El concepto de agencias privadas de protección ha sido usado por algunos libertarios (Rothbard, 2005; Hoppe, 1999) para ilustrar como podría ser la prestación de servicios de seguridad en una sociedad anarcocapitalista pero, salvo contadas excepciones (Hernandez, 2019), ha sido poco desarrollado. Es más, creemos que puede llevar a confusión al entenderse que la prestación de servicios de seguridad se circunscribiría en exclusiva a empresas privadas, con un diseño semejante al de las actuales organizaciones policiales o ejércitos estatales. Entendemos, al contrario, que en ese concepto se pueden incluir un conjunto muy plural de organizaciones. Este puede englobar desde empresas privadas de policías (Johnston, 1992) o mercenarios (Mandel, 2002; Stromberg, 2003) a hackers informáticos (Cruz, 2012) pasando por detectives privados (Horan, 1973) hasta agencias de recuperación de coches robados, y por supuesto grupos de vigilantes vecinales o grupos de autodefensa familiar sin ánimo lucrativo (Little y Sheffield, 1983). Cada uno con funciones de defensa y seguridad particulares pero al mismo tiempo con posibilidad de superponerse unos a otros. No vemos la necesidad de que exista un monopolio territorial de las mismas ni de que un individuo o comunidad tenga necesariamente porque contratar con una sola de ellas. De la misma forma que nuestra salud o vida puedan estar aseguradas con varias compañías al mismo tiempo (por ejemplo, podemos contar con un seguro de vida, con un seguro de viajes y con un seguro de automóvil que cubran prestaciones en parte similares) nada impide que una comunidad o individuo contraten con compañías distintas con prestaciones superpuestas entre sí. La República de Venecia, por ejemplo, acostumbrada a subcontratar servicios de defensa, no contrataba sólo con una compañía de mercenarios, sino con varias con funciones distintas (policía, defensa terrestre, defensa naval...) pero a su vez coincidentes en algunas funciones. De esta forma, no dependían sólo de una agencia que pudiese dominarles (Trease, 1985). En el estado moderno pueden coexistir en un mismo territorio varias organizaciones de defensa sin que exista ningún 
problema. Existen varias policías, públicas y privadas, o fuerzas armadas de distintas armas operando sobre un mismo espacio. De hecho, muchos gobiernos buscan también esta pluralidad para evitar que las policías o los ejércitos los dominen a ellos. A su vez, tampoco tiene porque darse un monopolio territorial de la defensa, de la misma forma que nuestros vecinos no tienen por qué contratar con la misma aseguradora de incendios. Es cierto que, a efectos de defensa, la contigüidad es algo importante, pero necesariamente en algún sitio tiene que ser delimitado donde acaba el espacio a proteger. De hecho, este fenómeno ya se da en el mundo estatal. El área de defensa de un estado (existen varios en el mundo, por lo tanto es obvio que no es un monopolio natural, pues de ser así existiría un sólo estado en el mundo) finaliza siempre en un punto, que es casi siempre contiguo al área de otro estado. Existen incluso en el mundo conurbaciones que pertenecen a dos o más unidades políticas, como en la frontera entre Flandes y Holanda, donde una casa pertenece y es defendida por un estado y la casa vecina por otro, o simplemente con cruzar la calle se pasa a la jurisdicción de otro estado (Hulsmann, 2003). También, dentro del territorio de muchos estados existen vacíos de soberanía como embajadas o bases militares de otros países. El estado sólo es monopolista, o pretende serlo, dentro de su propio dominio, el cual, a su vez, ha padecido a lo largo de su historia numerosas alteraciones de tamaño y forma, siendo fruto histórico de uniones y secesiones, de conquistas y de pérdidas territoriales. Muchas veces, de un estado han surgido varios, por lo que, si en lo que antes era uno, ahora existen cuatro o cinco (hay varios casos recientes en el este de Europa) no entendemos cual es la lógica que impera en este supuesto monopolio. El autor parece pensar en su análisis dentro de un marco estatista, en el sentido de que identifica a las agencias de seguridad como una suerte de estado, sólo que de propiedad privada, olvidando que incluso dentro del poder político han existido numerosas formas (Negro, 2010), algunas de ellas no soberanas territorialmente, y que lo que llamamos estado moderno no es más que el resultado del triunfo de una de esas formas, el estado nación soberano, tras cruentas luchas prolongadas durante siglos (Spruyt, 1994) y la progresiva desaparición, aunque no de forma total, de las mismas. 
Otra afirmación con la que no concordamos es la de que, hoy en día, todas las zonas habitadas de la tierra están sometidas a la jurisdicción de un estado, que el autor usa para justificar el supuesto triunfo final del estado y su inevitabilidad. En primer lugar, la mayor parte de la historia, hasta muy recientemente, ha transcurrido al margen de la dominación estatal. Numerosas porciones del planeta, en Asia, América, África y Oceanía no conocieron su existencia hasta los siglos XIX y XX. James Scott, (Scott, 2009), por ejemplo, documenta en sus escritos la existencia de un enorme territorio en el sudeste asiático, al que denomina Zomia, que vivió miles de años al margen del estado. Otra cuestión es que no existan registros históricos de los aconteceres de estos pueblos durante tanto tiempo, al no existir registros, y porque la historia como disciplina ha estado asociada en muchas ocasiones a la función de legitimadora del poder político, desconsiderando o acusando de bárbaros a los pueblos sin estados. Esta suerte de propaganda ha conseguido eliminar el recuerdo de las formas de vida preestatales, o en el mejor de los casos su recuerdo se circunscr ibe a leyendas o mitos de tradición oral.

En segundo lugar, no es cierto ni aún hoy, que todos los territorios de la tierra estén bajo jurisdicción estatal. Nominalmente es cierto que todos los territorios del planeta, excepto la Antártida, ven reconocida su pertenencia a algún estado reconocido por otros estados. Pero no es menos cierto que sobre muchos de ellos su jurisdicción es ficticia. Existen territorios en disputa, territorios dominados por guerrillas, narcos o piratas. Existen barrios o ciudades enteras, las llamadas ciudades ferales (Norton, 2003), en las que los aparatos y agentes del estado no tienen soberanía alguna, siendo dominados por otro tipo de actores sin que los detentadores de la soberanía legal puedan evitarlo. Estos territorios cuentan incluso con sistemas de defensa, seguridad y justicia extraestatales (Arias y Rodrigues, 2006). Estamos lejos aún de un dominio total de los estados sobre el planeta.

Nuestro autor tampoco aborda la cuestión de la escala sobre la que operarían las agencias. Los estados actualmente existentes no cuentan con una escala adecuada de funcionamiento, pues su actual forma deriva de azares históricos y no de un diseño empresarial pensado para la prestación de servicios públicos como los de 
defensa y seguridad. Existen estados reconocidos que cuentan con unos pocos miles de habitantes y otros con centenares en millones y que cuentan con formas geográficas muy disímiles (islas, enclaves, formas de paisaje irregulares). Una organización empresarial pensada para la prestación de servicios de este estilo modularía su prestación de acuerdo con las dinámicas de población o tecnologías. No es lo mismo atender una población de diez millones que de cien, ni es lo mismo defender un territorio con una tecnología que con otra. Una empresa comercial abandonaría territorios y se los dejaría a otras empresas, de cambiar alguno de estos factores, o bien podría ampliar su radio de acción, algo que un estado no puede hacer, como tampoco puede modular los servicios de seguridad de acuerdo con las preferencias relativas o las necesidades de las distintos grupos demográficos o de las distintas locaciones geográficas. Más bien tiene que pretender prestar un servicio homogéneo para todos y en todas partes, algo que además es de imposible cumplimiento, pues un servicio público nunca puede ser prestado de forma homogénea. Siempre tendrá que existir algún factor desigualador (como, por ejemplo, la distancia a las instalaciones de defensa o al armamento) dejando aparte que tal servicio sería percibido de forma muy desigual por la ciudadanía, constituyendo para algunos una bendición y para otros algo neutro o una maldición. No parece existir evidencia de que los de una determinada escala funcionen o cumplan sus funciones mejor que los de otra ni que se dé una tendencia clara hacia un estado único a escala mundial, ni tampoco forma de establecer un cálculo racional de cuál debería ser la escala correcta. No sucedería lo mismo con las agencias o unidades de protección privada. Como es obvio, éstas deberán contar con un ámbito o alcance de actuación, que puede ser desde una pequeña comunidad a un territorio de miles o centenares de miles de kilómetros cuadrados. La dimensión de tales agencias sería decidida empresarialmente, así como la forma en que se prestarán sus servicios o las funciones de protección que prestarán. Por lo tanto, por un lado es correcto que una agencia pudiera ser monopolista en un territorio dado, pero si no se define cual es la dimensión del territorio, que bien pudiera ser una vivienda, un condominio o un bloque de edificios, no vemos cual es la diferencia con una situación de anarquía ni el potencial 
peligro predador. Tampoco se define cual es el tiempo que tiene que durar el contrato con la agencia o agencias de seguridad para que estas puedan ser definidas como potenciales amenazas a la seguridad de sus contratantes. Es de prever que si estos perciben peligro de agresión por parte de esta rápidamente rompan el contrato y se aseguren en otra compañía.

Por otra parte, en la obra de Holcombe se puede percibir que su visión de una comunidad anarquista es la del territorio de un estado ya existente, pero sin gobierno. Una situación de anarquía no tiene por qué ser necesariamente así, pues la escala de organización humana probablemente sería muy distinta y organizada en todo tipo de comunidades y asociadas por pactos de muy diverso tipo. Los argumentos de Holcombe podrían ser plausibles partiendo de unas condiciones de inicio como las que él y otros libertarios plantean, pero no en organizaciones sociales distintas de las actuales. El nacionalismo metodológico impregna incluso a los libertarios, que plantean las organizaciones sociales del futuro análogas a las actuales sociedades-estado. Los modelos de análisis contemporáneos asocian ambas, de tal forma que, cuando, por ejemplo, nos referimos a la sociedad española queremos realmente decir la población que vive en el territorio del estado español, con indiferencia del tipo de relaciones que esta población mantenga entre sí o con otras sociedades. Al no existir una organización estatal, las formas de organización sociales estarían basadas en otras pautas de agregación y coordinación y, por tanto, los actuales esquemas mentales de lo que constituye una sociedad a defender es de presumir que no serían los mismos.

III

SOBRE LA POSIBILIDAD DE AGRESIÓN POR PARTE DE LAS AGENCIAS DE SEGURIDAD

El argumento sobre el que gira el trabajo de Holcombe es el de la posibilidad de que las agencias de seguridad privadas, una vez establecidas, descubran la posibilidad de poder extorsionar a sus clientes al encontrarse éstos sin medios de defensa. El autor apunta a una suerte de pulsión predatoria en este tipo organizaciones, 
que, aparentemente, no se daría o de darse sería fácilmente controlable en el resto de las unidades sociales y organizativas de la sociedad anárquica. Las empresas de seguridad contarían con una ventaja comparativa en el uso de la fuerza que les permitiría poder expoliar sin problema a sus clientes. Esta es la tesis central de este estudio, y por tanto merece ser minuciosamente diseccionada. En primer lugar, el profesor Holcombe tendría que indicarnos porque este tipo de propensión a la predación se daría especialmente entre las empresas de seguridad. Cómo bien apunta Walter Block (Block, 2005), de seguirse esa lógica en los restaurantes nos darían comida podrida para reducir costes, en los talleres de autos nos pondrían recambios en mal estado, o los bomberos provocarían incendios y los médicos enfermedades, por ejemplo, para obtener rentas. Así, de hecho, podría ocurrir en prácticamente cualquier trabajo, pues es muy fácil para un profesional ducho, si quisiese, el engañar o manipular a sus clientes para lucrarse. No hay razón para creer que estos empresarios tengan menor ánimo de lucro que los agentes de seguridad y si estos comportamientos no se dan en la mayoría de los sectores económicos, es de prever que tampoco se den en el ámbito de la seguridad, y por exactamente las mismas razones. La primera es que quedarían rápidamente sin clientes y, por tanto, se debilitaría su capacidad de dañar a su clientela al quedar sin recursos. En un entorno de libre mercado no parece ser frecuente que sean las empresas que ofrecen productos defectuosos o expolien a sus clientes con precios muy elevados o condiciones leoninas las que más crezcan o más beneficio obtengan. De hecho, este tipo de conductas sólo se dan en ámbitos monopolísticos ,y por tanto, debido a algún tipo de protección estatal. En un mundo sin estado este tipo de prácticas, si bien podrían existir, estarían condenadas a desaparecer desde sus comienzos. Repetimos lo anteriormente dicho, el modelo de Holcombe parece imaginar un territorio compacto protegido por una única agencia de seguridad privada, a imagen y semejanza de un estado, sólo que en este caso de propiedad privada. En un mundo con agencias de seguridad superpuestas o mezcladas en un territorio este fenómeno sería casi imposible que sucediese.

En segundo lugar, Holcombe presupone pasividad entre los clientes de la agencia. Esto es, presupone que éstos no van a reaccionar 
desde el principio a las extorsiones de la agencia y a intentar buscar respuestas. Una sociedad sin estado de estas características, por lógica, sólo podría darse en el futuro y después de una adopción consciente de tal modelo social. Es de presumir que en ese mundo existirá un porcentaje de personas conscientes de la naturaleza del estado y de los riesgos de su aparición. A diferencia de los pueblos preestatales, que no podían saber con exactitud lo que se les venía encima aunque lo intuían (Scott, 2003), una porción significativa de los habitantes de una futura sociedad anarquista es de suponer que estén familiarizados con los escritos de los autores anarcocapitalistas e incluso con los escritos del propio Holcombe en los que se advierte de la amenaza potencial de estas agencias, por lo que es de prever que estén alerta y adviertan desde el principio el peligro. De la misma forma en que no es posible sostener una sociedad comunista o una sociedad musulmana o cristiana sin que exista algún grupo significativo de comunistas, musulmanes o cristianos conscientes, lo mismo tendría necesariamente que acontecer en una sociedad libertaria. Una parte de la población bien en número o bien en dedicación a la causa, tendrán que ser libertarios conscientes, por lo menos en un número significativamente elevado (si no, ¿cómo se explicaría el haber llegado a esta situación?3) y por lo tanto tenderán a defender activamente sus libertades (Hummel, 2003). De esta forma, en el caso de detectar indicios de que la agencia de protección pudiera estar derivando hacia prácticas de extorsión, los clientes de la agencia podrían bien contratar otra $\mathrm{o}$, si esto no fuese suficiente, establecer estrategias de resistencia frente a la agencia agresora, de momento en fase larvada. También muchas otras organizaciones sociales, aun no siendo concebidas como agencias de protección, podrían convertirse en un contrapeso eficaz a las prácticas de la agencia agresora. Desde la autodefensa, a somatenes y vigilantes, iglesias o religiones organizadas o incluso agencias acostumbradas a asumir riesgos, como compañías de bomberos o de rescate, o incluso empresas de informática con capacidad de hackear la actuación de sus rivales, muchas serían, en una sociedad sin estado, las organizaciones con la potencialidad de organizar una defensa eficaz frente a un agresor de estas características. Los supuestos de Holcombe, como buena parte de los modelos de la elección pública, son ahistóricos, 
de tal forma que parece que no tuviesen historia o no fuesen capaz de aprender del pasado o simplemente incapaces de leer libros, aprender de ellos y explicárselos a los demás. Pero a su vez y contradiciendo su visión atemporal parece incurrir en una suerte de historicismo, como si existieran una suerte de leyes históricas que de forma necesaria impidan la creación de un tipo de sociedad de este tipo, simplemente porque el devenir histórico ha configurado una sociedad de corte estatal.

Por último, en tercer lugar, Holcombe presume que las agencias de seguridad son las únicas donde no se producirían comportamientos oportunistas y donde todos sus miembros obedecerían a sus jefes de forma mecánica, aun cuando las ordenes fuesen las de violar los derechos de propiedad del resto de la población. Es decir, supone que no sólo los jefes sino también los empleados de la organización estarían dispuestos a la agresión contra ciudadanos pacíficos sin oponer ningún tipo de resistencia a las órdenes de sus jefes (Cuzan, 1979). Una vez más, Holcombe, presupone rasgos de comportamiento predador a gran escala en sociedades en las cuales presumiblemente este comportamiento ya ha sido detectado y eliminado. Es más, supone que gente aparentemente tan malvada como los trabajadores de tales empresas dejarían a su jefe robar para él, y no se revolverían contra ellos para quitarles su parte. Es lugar común en la literatura de sucesos y un tema muy abordado en el cine y la literatura la situación en las cuales los bandidos o los mafiosos conspiran contra su jefe para ocupar su puesto o quitarle su botín. No sólo esto, los jefes de los estados con frecuencia tienen que afrontar rebeliones, pronunciamientos o golpes de estado por parte de sus subordinados, aun contando con el apoyo moral que implica la posesión de algún tipo de legitimidad de origen o de ejercicio (Luttwak, 2016). Holcombe parece también suponer que el único sitio donde las personas actuarían con lealtad al superior es precisamente una organización compuesta de hombres armados y entrenados para la lucha y, por lo que nuestro autor presupone, carentes de moral hasta tal grado que estuviesen dispuestos a agredir por dinero a personas pacíficas. Es también muy probable que, como también nos muestra la historia, este tipo de organizaciones se desbanden con frecuencia al verse privadas de dirección con cierto carisma y de una organización eficaz. Normalmente, tenían 
mucho más que temer los mercenarios que los reyes que los contrataban, mucho más duchos en intrigas y en artes políticas, pues sus jefes en muchas ocasiones terminaban sus días envenenados o estrangulados con la subsiguiente disolución de su banda (Trease, 1985).

Este fenómeno sería aún más acusado en el caso de empresas de este tipo, carentes de legitimidad. Es cierto que una agencia de protección del tipo de las planteadas por Holcombe puede tener una gran capacidad de ejercer fuerza física, y por tanto, capacidad de extorsionar, pero le falta un factor esencial, la aquiescencia al menos parcial del extorsionado, algo que a los estados les ha llevado mucho tiempo conseguir. De hecho, como indica Scott (Scott, 2003) el proceso de construcción de estados ha durado miles de años, con procesos de reversión a la no estatalidad incluidos, precisamente porque las agencias de violencia no conseguían vencer la resistencia, activa o pasiva, de las poblaciones que querían someter. Y esto era porque los dominados no veían como legítima la dominación e intentaban de todas las formas posibles sustraerse a ellas. Los gobernantes, que sabían bien esto, han buscado siempre contar con un aparato de legitimación (sacerdotes, historiadores, artistas, filósofos políticos...) que justificasen primero la necesidad y más recientemente la racionalidad de su dominio. Sin esta aquiescencia el dominio se realizaría por la mera fuerza física, algo que conduce necesariamente a una mayor resistencia, y por tanto a una menor capacidad de ejercer el poder. Lo que Holcombe insinúa es que la agencia sería capaz de dominar a la población y extorsionarla no sólo en ausencia de legitimidad de origen sino en medio de una población que contaría necesariamente con cierta consciencia anarquista. La experiencia histórica de los procesos de dominación nos muestra también que dominar a una población ya anarquista, como supone su modelo, es mucho más difícil que en situaciones en las que ya existe un estado (Keeley, 1996) pues el dominio de poblaciones muy disgregadas de forma muy dispersa es enormemente difícil en ausencia de una población conforme y de los instrumentos secundarios de regimentación de las poblaciones con que cuentan los estados ya instalados. Dominar pueblos como los apaches, aún con tecnología y ejércitos modernos, llevó cientos de años y enormes costes (Nevins, 2004). Holcombe pretende que una agencia extorsionadora se haría con el 
dominio de poblaciones anárquicas en un breve espacio de tiempo y sin resistencia de consideración. Cuando menos, esta afirmación merece ser puesta en cuarentena. Dominar un territorio ya estatalizado es relativamente fácil (Liberman, 1996) dado que la población ya está acostumbrada a obedecer y se cuentan con registros fiscales y censos, además de un aparato burocrático capaz de hacer obedecer las órdenes, y que normalmente pasa a obedecer al nuevo gobernante sin mayor dificultad, como se prueba en los numerosos casos de ocupación acontecidos en el último siglo. Basta con conseguir la obediencia de los aparatos centralizados. El problema para el nuevo ocupante que parta de una sociedad de anarquía previa es que carecería de todos esos instrumentos y sobre todo de un centro o capital desde la cual impartir órdenes al resto del territorio y sin el cual la agencia dominante tendría que ser capaz de desplegar su capacidad de coerción a pequeños grupos dispersos. Esta es la razón por la que la conquista de grandes imperios como el Inca o el Azteca se llevó a cabo en semanas, mientras que el dominio de apaches o mapuches requirió de centenares de años y muchos más costes. Casos como estos muestran que el imaginario de la anarquía manejado por muchos de sus críticos debería ser más elaborado si lo que se pretende es elaborar un modelo adecuado de su funcionamiento y no descansar en el modelo hobbesiano de anarquía del que no existen referentes históricos demostrables, esto es de una guerra de todos contra todos (Otterbein, 1997). Salvo, claro está, que entendamos por anarquía las situaciones creadas por guerras y saqueos fruto de la lucha por el poder entre distintos actores organizados políticamente, que es lo que Hobbes tenía en mente como espectador de las guerras civiles inglesas del siglo XVII. Una vez más, Holcombe nos muestra que su idea de una sociedad anárquica es la de un espacio estatal con una población estatalizada y organizada sobre la base de un estado previo, solo que por alguna extraña e inexplicada razón el estado ha desaparecido temporalmente de ella.

Mención aparte merece la cuestión de las mafias. Cierto es que los estados comparten buena parte de su estructura organizativa con las mafias (Tilly, 2007) y que, muy probablemente, hayan evolucionado a partir de estructuras de este tipo. Pero un estado es algo más que una mafia, pues cuenta con estructuras de legitimación mucho más sofisticadas (escuelas, religiones, arte estatal...) 
construidas a lo largo del tiempo, como bien ejemplifica Burckhardt en el capítulo "El estado como obra de arte" en su La cultura del renacimiento en Italia (Burckhardt, 1944). Por lo tanto, una mafia que partiese de una sociedad anarquista futura padecería las mismas limitaciones que cualquier otra agencia extorsionadora. El problema de Holcombe es, de nuevo, el partir de una visión contemporánea de las mafias, esto es, partir de la realidad de las modernas mafias estatalizadas y elaborar su modelo a partir de ellas. Las mafias modernas crecen en un ambiente estatista y obtienen sus rentas y su capacidad de acción bien de una débil estatalidad (Gambetta, 2007), no de una ausencia de esta, bien de operar en sectores que los estados regulan o prohíben (droga, juego, prostitución, urbanismo...). En el primer caso, los estados no son lo suficientemente fuertes como para dominar todo su territorio y por tanto impedir la existencia de agencias de protección fuera de su marco legal, pero si lo suficientemente fuertes como para condenar a la ilegalidad a cualquier alternativa privada y legítima que quisiera implantarse de forma pacífica y voluntaria. De esta forma, sólo aquellas personas ya predispuestas a quebrantar la legalidad prestarán servicios de protección, indistinguibles en muchos casos de la mera extorsión. En el segundo caso, el más común hoy en día, los estados actúan de hecho como subvencionadores de la actividad mafiosa (Thornton, 1991), al crear, mediante la prohibición, los nichos de mercado adecuados para que las mafias se desarrollen y garantizarles un precio mínimo para que sus actividades sean rentables. Queriendo, o sin querer, la regulación estatal fortalece a las mafias, de tal forma que no podríamos explicar sus actuales dimensiones sin entender que no dejan de ser apéndices parasitarios del estado, cuando no parte integrante del mismo, como acontece en los llamados narcoestados. Las mafias contemporáneas sin la ayuda no intencionada que el estado les presta no serían gran cosa, como se probó históricamente cuando el estado italiano en tiempos del fascismo decidió combatir de forma eficaz a estas organizaciones, que prácticamente desaparecieron. En cualquier caso, las mafias actualmente existentes y su evolución no constituyen un buen ejemplo para explicar la evolución estatal, dado que ésta se produce como resultado de una previa actuación estatal, en la que la mafia sólo sirve de eficaz colaboradora. La prueba está en 
que todas las mafias contemporáneas han existido o existen en un ambiente en el que el estado está de una forma $u$ otra presente.

IV

\section{¿ES INEVITABLE EL ESTADO?}

Holcombe afirma que la historia ha derrotado a la anarquía una vez y que presumiblemente seguirá haciéndolo en el futuro. Es una afirmación arriesgada. En primer lugar porque la mayor parte de la historia humana ha discurrido sin la presencia de entes estatales tanto en el tiempo como en el espacio (Johnson y Earle, 2003) y hasta aquí ha llegado la especie humana, por lo que se ve sin necesidad de su concurso. Los estados, por tanto, no han consolidado sino hasta hace muy poco tiempo su control del territorio, y aun así de forma nominal. Lo que acontece es que buena parte de los discursos históricos y geográficos se han elaborado alrededor de los estados y por consiguiente han eludido tratar lo que acontecía en espacios y tiempos ajenos a su existencia. Como antes apuntamos no existen relatos de cómo se organizaba la sociedad donde el estado no existía y por tanto el discurso de la historia parece confirmar la tesis de un incontenible desarrollo de las distintas formas de gobierno. En este relato no aparecen casi nunca vacíos históricos aestatales, y de existir se les etiqueta como siglos oscuros o eras o espacios bárbaros. Pero casi nunca se nos cuenta, por lo menos en las historias explicadas en los estudios obligatorios, que acontecía entonces allí.

Por otra parte, nuestro autor se refiere a los distintos grados de predación de las distintas formas estatales a lo largo de la historia. De ahí deduce la importancia de actuar preventivamente para lograr institucionalizar una forma de gobierno lo menos predatoria posible. Sin cuestionar que, en efecto, no todos los estados se comportan igual ni son igual de dañinos, convendría matizar algunos aspectos. El primero es que no por actuar preventivamente se puede evitar que un estado acabe actuando predatoriamente, como el propio autor reconoce. Estados diseñados con las máximas cautelas institucionales como los Estados Unidos se han convertido en organizaciones que se parecen bastante a lo que 
querían prevenir, como el propio Holcombe conoce bien (Holcombe, 2002). Si creamos un estado, esto es, un monopolista de la violencia, nada impedirá que acontezca lo que el propio autor estipula a respecto de las agencias de protección, pues se combinará una falta de capacidad de los ciudadanos para confrontar su poder con la existencia de una ideología que legitima tal actuación e incluso la ve como positiva y funcional para alcanzar la paz y la prosperidad. ¿Cómo oponerse a tal bendición? El segundo es la propia definición de predación y a quien se le aplica. Un estado puede ser predador, por ejemplo, en impuestos pero muy libre en otros ámbitos de la vida, como las libertades personales, o a la inversa. Otros pueden ser poco predadores dentro de sus fronteras pero muy predadores fuera de las mismas, y también a la inversa. También la forma de predación es importante a la hora de elaborar $\mathrm{u}$ diseño institucional que la minimice. Michael Mann (Mann, 1991) distingue dos tipos de configuración del poder. Una, a la que denomina poder despótico, referida a la arbitrariedad en el uso del poder, y otra, a la que se refiere con el término de poder infraestructural, que tiene que ver con la capacidad de un gobierno de penetrar las instituciones sociales y cambiar la vida o las condiciones de la vida de sus gobernados. Ambas usan el poder, la primera si cabe de forma más violenta que la segunda, la cual, al partir de una situación de aquiescencia mayor por parte de la población, no precisa de ejercer grandes dosis de violencia para conseguir sus objetivos. La segunda es capaz de obtener mayores cantidades de rentas y de obediencia que la primera, pero al ser menos violenta ¿podemos decir que es menos predatoria que la segunda? Un estado moderno es capaz de interferir, sin hacer uso de formas especialmente crueles de violencia, siempre teniendo presente que nunca se excluye del todo, (Huemer, 2013), hasta extremos que hace cien años serían visto como tiránicos. $Y$ viceversa, prácticas habituales incluso en estados limitados como los Estados Unidos de los padres fundadores, como la esclavitud o el exterminio de poblaciones indígenas, serían vistas hoy con horror. No es fácil determinar a priori, y sobre todo pensando en el futuro, cuales son o van a ser las posibles derivas de los estados, pues no olvidemos que están dirigidas por agentes conscientes y prestos a aprovechar cualquier oportunidad para expandir su poder, por ejemplo modificando el 
sentido de las normas e instituciones originarias. El ejemplo de la llamada interpretación viva de la Constitución, que propugna una aplicación de la misma no de forma literal sino "adaptada a los tiempos", podría servir de ejemplo de cómo instituciones en principio bien intencionadas acaban siendo deturpadas. El uso y abuso de una frase, en principio inocua, incorporada a la Constitución, el famoso principio de "promover el bienestar público" (Wagner, 1989) ha sido usada para crear los fundamentos del estado del bienestar norteamericano, que consume una parte ingente de las rentas de los estadounidenses. Por tanto, el diseño institucional precautorio podría prever y limitar algunos efectos futuros, pero no todos y sobre todo no podría evitar a medio plazo el desarrollo futuro de los estados hacia formas más predatorias, de éstos quererlo así. Tampoco podría impedirlo, porque no está definido en ninguna parte donde empieza y donde termina la frontera entre un estado predador y uno que no lo es, y definirla llevaría a debates que muy probablemente no condujesen a acuerdos aceptados por todos.

Defender y diseñar un estado mínimo no parece por tanto una solución satisfactoria para eliminar los potenciales efectos negativos de la instauración de un estado. Pudiera ser más lógica la solución establecida para intentar mitigar la esclavitud. Los que contra ella se rebelaron no lo hicieron para reducirla a un mínimo tolerable, o mejorar la condición de los esclavos. Los abolicionistas buscaron eliminarla por completo, y de esta forma consiguieron ambos objetivos. La lucha por una esclavitud mínima no acabaría con el problema sino que lo legitimaría.

\section{$\mathrm{V}$ \\ INCONSISTENCIAS Y CONTRADICCIONES EN LOS ARGUMENTOS DE HOLCOMBE.}

Los argumentos de Holcombe presentan además una serie de inconsistencias y contradicciones lógicas. En primer lugar, parece realizar un uso selectivo de la influencia de los incentivos de predadores y víctimas según esté intentando demostrar la imposibilidad de la anarquía o la factibilidad del estado mínimo. Así, cuando argumenta que la anarquía no es posible, lo hace centrándose en los incentivos de los 
depredadores y los ciudadanos, como ya hemos mencionado, son sólo víctimas pasivas incapaces de resistirse y cuyos incentivos no tienen importancia alguna. Los gobiernos son inevitables porque los predadores tienen incentivos para establecerlos y de este modo, así dice textualmente "La gente sin gobierno [...] se verá conquistada y dominada por bandas predatorias que establecerán un gobierno sobre ellas". (Holcombe, 2004, p. 334). Sin embargo, cuando argumenta que un estado mínimo es posible, de repente y sin explicación, los incentivos de los ciudadanos tienen importancia y pueden conducirles a modelar activamente el comportamiento de los predadores. Así, dice que "Los ciudadanos tienen un incentivo para crear y mantener preventivamente un gobierno que minimice la predación y que esté organizado para preservar, en la medida de los posible, la libertad de sus ciudadanos."(Holcombe, 2004, p. 335) Da la impresión de que Holcombe selecciona arbitrariamente, para apoyar el resultado que más le gusta, a quién, cómo y en qué momento afectan los incentivos al comportamiento.

Encontramos más problemas en el núcleo del argumento de Holcombe. Éste consiste en que la historia ha demostrado que la anarquía no sobrevive y que algunos gobiernos son menos predatorios que otros, por lo que la gente se puede beneficiar del establecimiento de un mecanismo institucional que impida que sean conquistados por una banda de predadores exteriores. Esto se consigue con la formación preventiva de un gobierno limitado en una forma que ellos y no los predadores determinen. El problema con este argumento es que si la historia ha demostrado que la anarquía no sobrevive, entonces es imposible crear un mecanismo institucional que prevenga que un grupo de ciudadanos sea conquistado por una banda predadora. Eso sólo lo podrían hacer partiendo de una situación de anarquía, pero él mismo ha afirmado que la anarquía no sobrevive. Según su propio argumento es imposible establecer preventivamente un gobierno porque esos ciudadanos ya han sido conquistados por una banda predadora.

La única posibilidad que permitiría la creación de un estado mínimo sería el establecimiento de tal sistema por los propios integrantes de la banda predadora, con independencia de los deseos e incentivos de los ciudadanos sometidos. Un vistazo a la historia permitiría comprobar si la tendencia de las bandas predadoras ha 
sido la de considerar el estado mínimo como la forma óptima de predación. Dada la dificultad de encontrar en la actualidad estados que, incluso desde una consideración generosa de lo que significa mínimo, cuadren en la categoría de estados mínimos, la respuesta es claramente negativa: la historia muestra que los estados mínimos no aparecen o no sobreviven.

Puede que lo que Holcombe esté diciendo, en realidad, es que es posible que los ciudadanos de un territorio derriben el gobierno y entonces, a partir de esa situación temporal de anarquía, formen preventivamente un estado limitado. Pero el problema con este argumento sería que entonces también sería posible establecer un sistema de anarquía sin necesidad de dar el paso de crear un estado limitado. Los ciudadanos simplemente tendrían que decidir convertir en permanente la situación temporal de anarquía. Si Holcombe afirmara que eso no sería posible porque entonces volverían a caer en las manos de una banda predadora, entonces tendría que explicar cómo fue posible en primer lugar derribar a la banda predadora que ya los dominaba. Si los ciudadanos pueden cooperar con éxito para derribar a una banda predadora que ya está en el poder, con más razón lo pueden hacer para resistirse a una banda predadora que no controla el territorio.

En su ansía por negar la posibilidad de la anarquía y afirmar la factibilidad del estado mínimo, Holcombe se ha acorralado a sí mismo. Sus propios argumentos llevan a concluir que, o bien la creación preventiva de un estado mínimo por parte de los ciudadanos es imposible, o bien que la anarquía es posible y, por tanto, el gobierno no es inevitable.

\section{VI CONCLUSIÓN}

Holcombe en su trabajo ha elaborado un modelo económico, eso sí, sin usar un lenguaje formal, sobre la imposibilidad de mantener en el tiempo una situación de anarquía, independientemente de que sea deseable o no. Este tipo de argumento ya había sido formalizado antes por teóricos de la elección pública como Winston Bush (Bush, 2005), de forma mucho más simplificada, y como todo modelo de 
este tipo parte de una serie de premisas, como la anarquía hobessiana o el pesimismo antropológico, caracterizado por la presunción de comportamientos no cooperativos entre los agentes con vista a maximizar su capacidad de obtener rentas. Pero en este tipo de modelos no encaja ni el papel de las ideas, ni la experiencia histórica real, y por lo tanto se invalidan buena parte de las conclusiones. Los supuestos previos del autor no se corresponden con la experiencia ni soportan un escrutinio por parte de disciplinas como la ciencia política o la sociología. El modelo puede ser muy elegante y refinado en términos formales pero al desconocer principios fundamentales del comportamiento social y, por lo que se puede ver en el texto, no desconocidos por el autor, el trabajo queda en un brillante, todo hay que decirlo, ejercicio teórico pero que no resuelve el viejo dilema de la posibilidad o imposibilidad de establecer una sociedad sin estado que permanezca estable en el tiempo.

\section{REFERENCIAS}

Arias,E.D. y Rodrigues, C.D. (2006), “The Myth of Personal Security: Criminal Gangs, dispute resolution and identity in Rio de Janeiro's favelas" en Latin America Politics $\mathcal{E}$ Society, vol. 48:4, pp. 53-81.

Block, Walter (2005), "Governmental Inevitability: Reply to Holcombe" en Journal of Libertarian Studies, vol. 19, n 3, Summer pp. 71-93.

Burckhardt, Jacob (1944), La cultura del renacimiento en Italia, Losada, Buenos Aires (e.o, 1860).

Bush, Winston (2005), "Individual Welfare in Anarchy" en Edward Stringham (ed.), Anarchy, State and Public Choice, Edward Elgar, Northampton, pp. 10-23.

Cruz Beltrán, José Luis (2012) “El perfil del nuevo analista de inteligencia del siglo XXI: De la inteligencia $<<$ tradicional $>>$ hacia la inteligencia económica; y outsourcing o externalización de los servicios de inteligencia" en Fernando Velasco y Rubén Arcos (eds.) Cultura de Inteligencia: Un elemento para la reflexión y colaboración internacional, Plaza y Valdés, Madrid, pp. 245-273.

Cuzan, Alfred G. (1979), "Do We Ever Really Get Out of Anarchy" en Journal of Libertarian Studies, vol 3, n 2, pp. 151-158. 
Gambetta, Diego (2007), La mafia siciliana: El negocio de la protección privada,Fondo de Cultura Económica, México.

Hernandez Cabrera, José Francisco (2019), Defensa y seguridad: ¿Estatal o privada?, Unión editorial, Madrid.

Holcombe, Randall G. (1995), Public Policy and the Quality of Life: Market Incentives versus Government Planning, Greenwood Press, Westport.

- (2002), From Liberty to Democracy. The Transformation of American Government, University of Michigan Press, AnnArbor.

- (2004), "Government: Unnecessary but Inevitable" en Independent Review vol. VIII, n 3, Winter, pp. 325-342.

- (2005) "Is Government Inevitable? Reply to Leeson and Stringham" en Independent Review, vol. IX, no 4, Spring, pp. 551-557.

- (2008) , "Why does Government Produce National Defense" en Public Choice, no 137, pp. 11-19.

Hoppe, Hans Hermann (1999), "The Private Production of Defense" en Journal of Libertarian Studies, vol. 14, $\mathrm{n}^{\circ}$ 1, winter, pp. 27-52.

Horan, J.D. (1973), Los Pinkerton, Bruguera, Barcelona.

Huemer, Michael (2013), The problem of political authority: an examination of the right to coerce and the duty to obey, Palgrave Macmillan, Basingtoke.

Hulsmann, Jorg Guido (2003), "Secession and the Production of Defense" en Hans-Hermann Hoppe (ed.), The Myth of National Defense, Ludwig von Mises Institute, Auburn, pp. 369-413.

Hummel, Jeffrey Rogers (2003), “The Will to be Free: The Role of Ideology in National Defense" en Hans-Hermann Hoppe (ed.), The Myth of National Defense. Essays on the Theory and History of Security Production, Ludwig von Mises Institute, Auburn 275-297. Johnson, Allen W y Earle, Timothy (2003), La evolución de las sociedades humanas, Ariel, Barcelona.

Johnston, Les, (1992) The Rebirth of Private Policing, Routledge, London.

Keeley, Lawrence H. (1996), War before Civilization: The Myth of the Peaceful Savage, Oxford University Press, Oxford.

Liberman, Peter (1996), Does Conquest Pay? The Explotaition of Occupied Industrial Society, Princeton University Press, Princeton.

Little, Craig B. y Sheffield, Christopher P. (1983) "Frontiers and Criminal Justice: English Private Prosecution Societies and 
American Vigilantism in the Eighteen and Nineteen Centuries" en American Sociological Review, vol. 48, n 6, December, pp. 796-808.

Luttwak, Edward N. (2016), Coup d'Etat: A Practical Handbook, Harvard University Press, Cambridge.

Mandel, Robert (2002) Armies without States. The Privatization of Security, Lynne Riener Publishers, Boulder.

Mann, Michael (1991), Las fuentes del poder social. Vol. 1: Una historia del poder desde los comienzos hasta 1760 d.C, Alianza editorial, Madrid.

Negro Pavón, Dalmacio (2010), Historia de las formas del Estado, El Buey Mudo, Madrid.

Neocleous, Mark (2003), Imagining the State, Open University Press, Maidenhead.

Nevins, Thomas J. (2004), "Introduction" en Helge Ingstad, The Apache Indians. In Search of the Missing Tribe, University of Nebraska Press, Lincoln, pp. xvii-xxxix.

Norton, Richard J. (2003), "Feral Cities" en Naval War College Review, vol. LVI, n 4, Autumn, pp. 97-106.

Otterbein, Keith F. (1997), "The Origins of War" en Critical Review, vol. 11, n 2, Spring, pp. 251-277.

Powell, Benjamin y Coyne, Christopher (2003), “Do Pessimistic Assumptions about Human Behavior Justify Government?" en Journal of Libertarian Studies, vol. 17, n $\mathrm{n}^{\circ}$, Fall, pp. 17-37.

Rothbard, Murray N. (2005), Hacia una nueva libertad, Grito Sagrado, Buenos Aires.

Scott, James C. (2003), Los dominados y el arte de la resistencia, Txalaparta, Tafalla.

- (2009). The Art of Not Being Governed: An Anarchist History of Upland Southeast Asia, Yale University Press. New Haven.

Spruyt, Hendrik (1994), The Sovereign State and Its Competitors: An Analysis of System Change, Princeton, Princeton University Press. Stromberg, Joseph (2003) "Mercenaries, Guerrillas, Militias, and the Defense of Minimal States and Free Societies" en Hans-Hermann Hoppe (edit.), The Myth of National Defense, Ludwig von Mises Institute, Auburn, pp. 215-238.

Tilly, Charles (2007), "Guerra y construcción del estado como crimen organizado" en Relaciones Internacionales, n 5, pp. 1-26. 
116 MIGUEL ANXO BASTOS BOUBETA Y ÓSCAR RODRÍGUEZ CARREIRO

Thornton, Mark (1991), The Economics of Prohibition, University of Utah Press, Salt Lake City.

Trease, Geoffrey (1985), Los condotieros, soldados de fortuna, Orbis, Barcelona.

Wagner, Richard E. (1989), To Promote General Welfare: Market Processes VS. Political Transfers, Pacific Research Institute for Public Policy, San Francisco. 